\title{
Spontaneous reduction of the heat conductivity by a temperature gradient-driven instability in electron-ion plasmas
}

\author{
Makoto Hattori and Keiichi Umetsu \\ Astronomical Institute, Tôhoku University, Aoba, Sendai 980-8578, Japan
}

(Received June 21, 2000; Revised February 21, 2001; Accepted February 21, 2001)

\begin{abstract}
We have shown that there exist low-frequency growing modes driven by a global temperature gradient in electron and ion plasmas, by linear perturbation analysis within the frame work of plasma kinetic theory. The driving force of the instability is the local deviation of the distribution function from the Maxwell-Boltzmann due to a global temperature gradient. Our results suggest that the realization of the global thermal equilibrium is postponed by the local instability which is induced for quicker realization of local thermal equilibrium state in plasmas. The instability provides a new possibility to create and amplify cosmic magnetic fields without the seed for a magnetic field.
\end{abstract}

\section{Introduction}

Ramani and Laval (1978) found a new plasma instability which may contribute to the reduction of the electron mean free path, henceforce the reduction of the heat conduction. They showed that the temperature gradient leads to an anisotropic electron velocity distribution function and that the anisotropy of the velocity distribution function drives an instability like the Weibel instability (Weibel, 1959; Fried, 1959; Melrose, 1986). They proposed that the chaotic magnetic and electric fields, induced by the instability, scatter the electrons, so that the electron mean free path is reduced by many orders of magnitude. However, the instability found by Ramani and Laval fails in reducing the electron mean free path because of the following reasons. Since Ramani and Laval assumed that only electrons respond to the mode and ions were treated as fixed back-ground particles, the phase velocity of the wave must be faster than the ion sound velocity $v_{\mathrm{th}, i}=\sqrt{2 k_{\mathrm{B}} T_{i} / m_{i}}$, where $T_{i}, n_{i}$, and $m_{i}$ are the ion temperature, ion number density, and ion mass, respectively. In the astrophysical plasma, the ion mass can be safely replaced by the proton mass. The unstable mode found by Ramani and Laval (1978) has non zero real part of the wave frequency with a phase velocity of $\sim \epsilon v_{\mathrm{th}, e}, \epsilon<1$. Therefore, the application limit of their analysis sets a relatively high lower limit on $\epsilon$ as $\epsilon>\sqrt{m_{e} / m_{i}} \sim 0.025$ when $T_{i}=T_{e}$ (hereafter we refer to this lower limit as the wall of the square root of the mass ratio). To explain the two-phase nature of the hot gas in the cluster central region (Ikebe et al., 1999), the instability found by them is not useful and a mechanism which can break the wall of the square root of the mass ratio is required.

In this paper, we extend the Ramani and Laval (1978) analysis including the response of ions to examine whether we can break the wall of the square root of the mass ratio. We

Copy right $(\mathrm{C})$ The Society of Geomagnetism and Earth, Planetary and Space Science (SGEPSS); The Seismological Society of Japan; The Volcanological Society of Japan; The Geodetic Society of Japan; The Japanese Society for Planetary Sciences. limit our attention to pressure equilibrium; in other words, the scale of the interface is much smaller than the pressure scale height. Then, the gravitational force can be neglected. The main results of this paper were reported in Hattori and Umetsu (2000).

\section{Distribution Function}

When $\epsilon<1$, the discussion given in the previous section ensures that the solution of the Boltzmann equation of electron and ion can be expanded in powers of $\epsilon$ (Chapman and Cowling, 1960)

$$
\begin{aligned}
f_{e} & =f_{\mathrm{m}, e}+f_{e}^{(1)}+f_{e}^{(2)}+\cdots, \\
f_{i} & =f_{\mathrm{m}, i}+f_{i}^{(1)}+f_{i}^{(2)}+\cdots,
\end{aligned}
$$

where $f_{\mathrm{m}, e}$ and $f_{\mathrm{m}, i}$ are Maxwellian distribution functions for electrons and ions, respectively; $f_{e}^{(k)}$ and $f_{i}^{(k)}(k=1,2, \ldots)$ describe the deviation of the distribution functions from the Maxwellian to order $\epsilon^{k}$. Since we assume that $T_{i}(x)=$ $T_{e}(x) \equiv T(x)$ and charge neutrality must be maintained, the Maxwellian parts for both electrons and ions are written as $f_{\mathrm{m}, e}=n_{0}(x)\left[\pi v_{\mathrm{th}, e}(x)\right]^{-3 / 2} \exp \left\{-\left[v / v_{\mathrm{th}, e}(x)\right]^{2}\right\}$ and $f_{\mathrm{m}, i}=n_{0}(x)\left[\pi v_{\mathrm{th}, i}(x)\right]^{-3 / 2} \exp \left\{-\left[v / v_{\mathrm{th}, i}(x)\right]^{2}\right\}$, respectively. Here $n_{0}(x)$ is the electron number density, $v_{\mathrm{th}, e}(x) \equiv$ $\sqrt{2 k_{\mathrm{B}} T(x) / m_{e}}$, and $v_{\mathrm{th}, i}(x) \equiv \sqrt{2 k_{\mathrm{B}} T(x) / m_{i}}$.

The pressure equilibrium assumption gives

$$
\frac{\nabla n_{0}}{n_{0}}=-\frac{\nabla T}{T}=-\frac{1}{L} \delta_{T} .
$$

Once this condition is satisfied, the pressure is time independent everywhere, even if a secular variation of the temperature due to thermal conduction is taken into account. Then the time dependence of the density can be related to that of the temperature as

$$
\frac{1}{n_{0}} \frac{\partial n_{0}}{\partial t}=-\frac{1}{T} \frac{\partial T}{\partial t}
$$


The Boltzmann equation under the pressure equilibrium condition without background electric and magnetic fields leads to

$$
\frac{\partial f_{e}}{\partial t}+v_{x} \frac{\partial f_{e}}{\partial x}=\left(\frac{\partial f_{e}}{\partial t}\right)_{\mathrm{c}},
$$

where $\left(\partial f_{e} / \partial t\right)_{\mathrm{c}}$ is the collision term and the equilibrium electric field does not appear because of the pressure equilibrium condition. The secular time variation of the distribution function is ascribed to the secular variation of the temperature due to thermal conduction. Since the cool region with temperature $T_{1}$ is considered to be immersed in a heat bath of a temperature $T_{2}$, the temperature of the interface increases monotonically owing to heat conduction. Hence, the time evolution of temperature can be described as

$$
\frac{\partial T}{\partial t}=\epsilon \delta_{T} \frac{v_{\mathrm{th}, e}}{L} T
$$

Then,

$$
\left|\frac{\partial f_{e}}{\partial t}\right| \sim \frac{1}{T}\left|\frac{\partial T}{\partial t}\right| f_{e} \sim \epsilon \delta_{T} \frac{v_{\mathrm{th}, e}}{L} f_{e} .
$$

The simplest choice of the collision term is the Krook operator, such that

$$
\left(\frac{\partial f_{e}}{\partial t}\right)_{\mathrm{c}}=-v_{e}\left(f_{e}-f_{\mathrm{m}, e}\right),
$$

where $v_{e}=v_{\text {th,e }} / \lambda_{e}$ is the collision frequency assumed to be constant. Substituting the collision term by the Krook operator, ordering the Boltzmann equation in $\epsilon^{1}$ and $\epsilon^{2}$ provides

$$
\begin{aligned}
f_{e}^{(1)} & =-\frac{1}{v_{e}} v_{x} \frac{\partial f_{\mathrm{m}, e}}{\partial x}, \\
f_{e}^{(2)} & =-\frac{1}{v_{e}}\left(\frac{\partial f_{\mathrm{m}, e}}{\partial t}+v_{x} \frac{\partial f_{e}^{(1)}}{\partial x}\right) .
\end{aligned}
$$

It shows that the system can be treated as steady state in the first order of $\epsilon$ but the secular variation cannot be neglected in second order of $\epsilon$. The time derivative of the MaxwellBoltzmann distribution function can be described as

$$
\begin{aligned}
\frac{\partial f_{\mathrm{m}, e}}{\partial t} & =-\frac{1}{2}\left(5-\frac{2 v^{2}}{v_{\mathrm{th}, e}^{2}}\right) \frac{1}{T} \frac{\partial T}{\partial t} f_{\mathrm{m}, e} \\
& =-\frac{\epsilon \delta_{T}}{2} \frac{v_{\mathrm{th}, \mathrm{e}}}{L}\left(5-\frac{2 v^{2}}{v_{\mathrm{th}, e}^{2}}\right) f_{\mathrm{m}, e} .
\end{aligned}
$$

The first- and the second-order solutions for the electron distribution function are thus obtained as follows:

$$
\begin{aligned}
& f_{e}^{(1)}=\frac{\epsilon \delta_{T}}{2} \frac{v_{x}}{v_{\mathrm{th}, e}}\left(5-\frac{m_{e} v^{2}}{k_{\mathrm{B}} T}\right) f_{\mathrm{m}, e}, \\
& f_{e}^{(2)}=\frac{\epsilon^{2} \delta_{T}}{2}\left[\left(5-\frac{2 v^{2}}{v_{\mathrm{th}, e}^{2}}\right)+\frac{v_{x}^{2}}{v_{\mathrm{th}, e}^{2}}\left(5-\frac{4 v^{2}}{v_{\mathrm{th}, e}^{2}}\right)\right. \\
& \left.+\frac{v_{x}^{2}}{2 v_{\mathrm{th}, e}^{2}}\left(5-\frac{2 v^{2}}{v_{\mathrm{th}, e}^{2}}\right)^{2}\right] f_{\mathrm{m}, e},
\end{aligned}
$$

where we have neglected the second derivative of $T(x)$ with respect to $x$ in deriving the second-order solution.
These equations show that the anisotropy in the velocity distribution functions is induced by the temperature gradient. The first-order solution is an odd function of the velocity. On the other hand, the second-order solution is even in the velocity. It is straightforward to as sure that the zero background electric field assumption is consistent with the zero electric current condition under the pressure equilibrium plasma condition, by checking $\left\langle f_{e} \vec{v}\right\rangle=\overrightarrow{0}$, where \langle\rangle denotes the average over the velocity.

Substituting $m_{e}$ for $m_{i}$ and $v_{e}$ to $v_{i}=v_{i} / \lambda_{i}$ in Eqs. (12) and (13), the first and the second order distribution functions $f_{i}^{(1)}$ and $f_{i}^{(2)}$ for ions are obtained.

\section{Dispersion Relation}

In this paper, perturbations with a short-wavelength wave are treated, such that

$$
\lambda \ll \lambda_{e}
$$

Therefore, the collision term in the Boltzmann equation for the perturbed variables can be neglected. In the short-wavelength limit, the collisionless Boltzmann equation is a good representative for the evolution of the perturbed distribution functions, and the plain wave $\exp [i(\vec{k} \cdot \vec{r}-\omega t)]$ is a good representative for the perturbed quantities (e.g., electron distribution function as $\delta f_{e}=f_{\vec{k}, e} \exp [i(\vec{k} \cdot \vec{r}-\omega t)]$, electric field as $\vec{E}=\vec{E}_{\vec{k}} \exp [i(\vec{k} \cdot \vec{r}-\omega t)]$, and magnetic field as $\left.\vec{B}=\vec{B}_{\vec{k}} \exp [i(\vec{k} \cdot \vec{r}-\omega t)]\right)$. From the linearized Boltzmann equation in terms of the perturbed quantities of both electrons and ions, the equations for the perturbed distribution functions of electrons and ions are obtained as

$$
\begin{aligned}
-i(\omega & -\vec{k} \cdot \vec{v}) f_{\vec{k}, e} \\
& =+\frac{e}{m_{e}}\left(\vec{E}_{\vec{k}}+\vec{v} \times \frac{\vec{B}_{\vec{k}}}{c}\right) \cdot\left(\frac{\partial f_{e}}{\partial \vec{v}}\right) \\
-i(\omega & -\vec{k} \cdot \vec{v}) f_{\vec{k}, i} \\
& =-\frac{e}{m_{i}}\left(\vec{E}_{\vec{k}}+\vec{v} \times \frac{\vec{B}_{\vec{k}}}{c}\right) \cdot\left(\frac{\partial f_{i}}{\partial \vec{v}}\right) .
\end{aligned}
$$

As shown below, the low-frequency condition requires that the charge density be small, implying that the electric current has to be almost transverse for purely electronic oscillations. Therefore, only two modes are expected. Without loss of generality, the wave vector $\vec{k}$ can be taken in the $x-y$ plane as $k_{x}=k \cos \theta, k_{y}=k \sin \theta$, and $k_{z}=0$. We expect one mode with the magnetic field along the $z$-direction (mode (1): $B_{x}=$ $B_{y}=0$ and $\left.B_{z} \neq 0\right)$, and the other one with the magnetic field in the $x-y$ plane (mode (2): $B_{x}, B_{y} \neq 0$ and $B_{z}=0$ ). We thus denote the component of a vector along the direction of the wave vector by a $k$-subscript and the component along the perpendicular direction of the wave vector in the $x-y$ plain by a $\perp$-subscript. The $x$-component of velocity, for example, can be expressed as $v_{x}=v_{k} \cos \theta-v_{\perp} \sin \theta$ in terms of $v_{k}$ and $v_{\perp}$.

Faraday's law leads to $E_{z}=0$ (i.e., $\vec{E}_{\vec{k}}=\left(E_{k}, E_{\perp}, 0\right)$ ) and $k E_{\perp}=(\omega / c) B_{z}$ for mode (1). For mode (2), $\operatorname{div} \vec{B}=0$ yields $B_{k}=0$ (i.e., $\left.\vec{B}_{\vec{k}}=\left(0, B_{\perp}, 0\right)\right)$, and Faraday's law and $B_{z}=0$ lead to $E_{\perp}=0$ and $k E_{z}=-(\omega / c) B_{\perp}$. Then we 
obtain

$$
\begin{aligned}
&(\vec{k} \cdot \vec{v}-\omega) f_{\vec{k}, e}=-\frac{i e}{m_{e}} {\left[\left(E_{k}+\frac{v_{\perp}}{c} B_{z}\right) \frac{\partial f_{e}}{\partial v_{k}}\right.} \\
&\left.+\left(E_{\perp}-\frac{v_{k}}{c} B_{z}\right) \frac{\partial f_{e}}{\partial v_{\perp}}\right], \\
&(\vec{k} \cdot \vec{v}-\omega) f_{\vec{k}, i}=+\frac{i e}{m_{i}}\left[\left(E_{k}+\frac{v_{\perp}}{c} B_{z}\right) \frac{\partial f_{i}}{\partial v_{k}}\right. \\
&\left.+\left(E_{\perp}-\frac{v_{k}}{c} B_{z}\right) \frac{\partial f_{i}}{\partial v_{\perp}}\right]
\end{aligned}
$$

for mode (1), and

$$
\begin{aligned}
(\vec{k} \cdot \vec{v}-\omega) f_{\vec{k}, e}=-\frac{i e}{m_{e}} & {\left[\left(E_{k}-\frac{v_{z}}{c} B_{\perp}\right) \frac{\partial f_{e}}{\partial v_{k}}\right.} \\
+ & \left.\left(E_{z}+\frac{v_{k}}{c} B_{\perp}\right) \frac{\partial f_{e}}{\partial v_{z}}\right], \\
(\vec{k} \cdot \vec{v}-\omega) f_{\vec{k}, i}=+\frac{i e}{m_{i}} & {\left[\left(E_{k}-\frac{v_{z}}{c} B_{\perp}\right) \frac{\partial f_{i}}{\partial v_{k}}\right.} \\
& \left.+\left(E_{z}+\frac{v_{k}}{c} B_{\perp}\right) \frac{\partial f_{i}}{\partial v_{z}}\right]
\end{aligned}
$$

for mode (2).

To first order in $\omega /\left(k v_{\text {th }, e}\right)$ and $\omega /\left(k v_{\text {th }, i}\right),(\vec{k} \cdot \vec{v}-\omega)^{-1}=$ $(1 / k) P\left(1 / v_{k}\right)+\left(\omega / k^{2}\right) P\left(1 / v_{k}^{2}\right)+i(\pi / k) \delta\left(v_{k}\right)-i(\pi / k)$. $(\omega / k)\left(d \delta\left(v_{k}\right) / d v_{k}\right)$, where $P$ denotes the principal value and the signs in front of the delta functions reflect the causality condition. Then, with the help of Faraday's law, Eqs. (17) and (18) yield

$$
\begin{aligned}
f_{\vec{k}, e}= & -\frac{i e}{m_{e}}\left\{\left[\frac{1}{k} P \frac{1}{v_{k}}+\frac{\omega}{k^{2}} P \frac{1}{v_{k}^{2}}+\frac{i \pi}{k} \delta\left(v_{k}\right)\right.\right. \\
& \left.-\frac{i \pi \omega}{k^{2}} \frac{d \delta\left(v_{k}\right)}{d v_{k}}\right]\left(E_{k}+\frac{v_{\perp}}{c} B_{z}\right) \\
& \times\left(-\frac{2 v_{k}}{v_{\mathrm{th}, e}^{2}}+\frac{\epsilon \delta_{T}}{2 v_{\mathrm{th}, e}}\left[\cos \theta\left(5-\frac{2 v^{2}}{v_{\mathrm{th}, e}^{2}}\right)\right.\right. \\
& \left.\left.-\frac{2 v_{k}}{v_{\mathrm{th}, e}^{2}}\left(v_{k} \cos \theta-v_{\perp} \sin \theta\right)\left(7-\frac{2 v^{2}}{v_{\mathrm{th}, e}^{2}}\right)\right]\right) \\
& -\frac{B_{z}}{c k}\left(-\frac{2 v_{\perp}}{v_{\mathrm{th}, e}^{2}}+\frac{\epsilon \delta_{T}}{2 v_{\mathrm{th}, e}}\left[-\sin \theta\left(5-\frac{2 v^{2}}{v_{\mathrm{th}, e}^{2}}\right)\right.\right. \\
& \left.\left.\left.-\frac{2 v_{\perp}}{v_{\mathrm{th}, e}^{2}}\left(v_{k} \cos \theta-v_{\perp} \sin \theta\right)\left(7-\frac{2 v^{2}}{v_{\mathrm{th}, e}^{2}}\right)\right]\right)\right\} f_{\mathrm{m}, e},
\end{aligned}
$$

$$
\begin{aligned}
f_{\vec{k}, i}= & +\frac{i e}{m_{e}}\left\{\left[\frac{1}{k} P \frac{1}{v_{k}}+\frac{\omega}{k^{2}} P \frac{1}{v_{k}^{2}}+\frac{i \pi}{k} \delta\left(v_{k}\right)\right.\right. \\
& \left.-\frac{i \pi \omega}{k^{2}} \frac{d \delta\left(v_{k}\right)}{d v_{k}}\right]\left(E_{k}+\frac{v_{\perp}}{c} B_{z}\right) \\
& \times\left(-\frac{2 v_{k}}{v_{\mathrm{th}, i}^{2}}+\frac{\epsilon \delta_{T}}{2 v_{\mathrm{th}, i}}\left[\cos \theta\left(5-\frac{2 v^{2}}{v_{\mathrm{th}, i}^{2}}\right)\right.\right. \\
& \left.\left.-\frac{2 v_{k}}{v_{\mathrm{th}, i}^{2}}\left(v_{k} \cos \theta-v_{\perp} \sin \theta\right)\left(7-\frac{2 v^{2}}{v_{\mathrm{th}, i}^{2}}\right)\right]\right) \\
& -\frac{B_{z}}{c k}\left(-\frac{2 v_{\perp}}{v_{\mathrm{th}, i}^{2}}+\frac{\epsilon \delta_{T}}{2 v_{\mathrm{th}, i}}\left[-\sin \theta\left(5-\frac{2 v^{2}}{v_{\mathrm{th}, i}^{2}}\right)\right.\right.
\end{aligned}
$$

$$
\left.\left.\left.-\frac{2 v_{\perp}}{v_{\mathrm{th}, i}^{2}}\left(v_{k} \cos \theta-v_{\perp} \sin \theta\right)\left(7-\frac{2 v^{2}}{v_{\mathrm{th}, i}^{2}}\right)\right]\right)\right\} f_{\mathrm{m}, i}
$$

for mode (1). Similarly, from Eqs. (19) and (20), we obtain

$$
\begin{aligned}
& f_{\vec{k}, e}=-\frac{i e}{m_{e}}\left\{\left[\frac{1}{k} P \frac{1}{v_{k}}+\frac{\omega}{k^{2}} P \frac{1}{v_{k}^{2}}+\frac{i \pi}{k} \delta\left(v_{k}\right)\right.\right. \\
& \left.-\frac{i \pi \omega}{k^{2}} \frac{d \delta\left(v_{k}\right)}{d v_{k}}\right]\left(-\frac{v_{z}}{c} B_{\perp}\right) \\
& \times\left(-\frac{2 v_{k}}{v_{\mathrm{th}, e}^{2}}+\frac{\epsilon \delta_{T}}{2 v_{\mathrm{th}, e}}\left[\cos \theta\left(5-\frac{2 v^{2}}{v_{\mathrm{th}, e}^{2}}\right)\right.\right. \\
& \left.\left.-\frac{2 v_{k}}{v_{\mathrm{th}, e}^{2}}\left(v_{k} \cos \theta-v_{\perp} \sin \theta\right)\left(7-\frac{2 v^{2}}{v_{\mathrm{th}, e}^{2}}\right)\right]\right) \\
& +\frac{B_{\perp}}{c k}\left[-\frac{2 v_{z}}{v_{\mathrm{th}, e}^{2}}-\frac{\epsilon \delta_{T}}{2 v_{\mathrm{th}, e}}\left(v_{k} \cos \theta-v_{\perp} \sin \theta\right)\right. \\
& \left.\left.\times\left(7-\frac{2 v^{2}}{v_{\mathrm{th}, e}^{2}}\right)\right]\right\} f_{\mathrm{m}, e} \\
& f_{\vec{k}, i}=+\frac{i e}{m_{i}}\left\{\left[\frac{1}{k} P \frac{1}{v_{k}}+\frac{\omega}{k^{2}} P \frac{1}{v_{k}^{2}}+\frac{i \pi}{k} \delta\left(v_{k}\right)\right.\right. \\
& \left.-\frac{i \pi \omega}{k^{2}} \frac{d \delta\left(v_{k}\right)}{d v_{k}}\right]\left(-\frac{v_{z}}{c} B_{\perp}\right) \\
& \times\left(-\frac{2 v_{k}}{v_{\mathrm{th}, i}^{2}}+\frac{\epsilon \delta_{T}}{2 v_{\mathrm{th}, i}}\left[\cos \theta\left(5-\frac{2 v^{2}}{v_{\mathrm{th}, i}^{2}}\right)\right.\right. \\
& \left.\left.-\frac{2 v_{k}}{v_{\mathrm{th}, i}^{2}}\left(v_{k} \cos \theta-v_{\perp} \sin \theta\right)\left(7-\frac{2 v^{2}}{v_{\mathrm{th}, i}^{2}}\right)\right]\right) \\
& +\frac{B_{\perp}}{c k}\left[-\frac{2 v_{z}}{v_{\mathrm{th}, i}^{2}}-\frac{\epsilon \delta_{T}}{2 v_{\mathrm{th}, e}}\left(v_{k} \cos \theta-v_{\perp} \sin \theta\right)\right. \\
& \left.\left.\times\left(7-\frac{2 v^{2}}{v_{\mathrm{th}, i}^{2}}\right)\right]\right\} f_{\mathrm{m}, i}
\end{aligned}
$$

for mode (2).

Poisson's equation is written as

$$
i k E_{k}=4 \pi e\left(\left\langle f_{\vec{k}, i}\right\rangle-\left\langle f_{\vec{k}, e}\right\rangle\right) .
$$

Keeping only the first non-vanishing order in $\epsilon$ for mode (1), each term on the right-hand side provides

$$
\begin{aligned}
& -4 \pi e\left\langle f_{\vec{k}, e}\right\rangle=-\frac{i}{k}\left[\frac{2}{\lambda_{\mathrm{D}}^{2}} E_{k}-\epsilon \delta_{T} \frac{\omega_{\mathrm{pe}}^{2}}{c v_{\mathrm{th}, e}} B_{z} \sin \theta\right], \\
& +4 \pi e\left\langle f_{\vec{k}, i}\right\rangle=-\frac{i}{k}\left[\frac{2}{\lambda_{\mathrm{D}}^{2}} E_{k}-\epsilon \delta_{T} \frac{\omega_{\mathrm{pi}}^{2}}{c v_{\mathrm{th}, i}} B_{z} \sin \theta\right],
\end{aligned}
$$

where $\omega_{\text {pe }}$ and $\omega_{\text {pi }}$ are the electron and ion plasma frequencies, defined by $\omega_{\mathrm{pe}} \equiv \sqrt{\left(4 \pi n_{0} e^{2} / m_{e}\right)}$ and $\omega_{\mathrm{pi}} \equiv$ $\sqrt{\left(4 \pi n_{0} e^{2} / m_{i}\right)}$, respectively; $\lambda_{\mathrm{D}} \equiv \sqrt{k_{\mathrm{B}} T /\left(4 \pi n_{0} e^{2}\right)}$ is the Debye length. It is trivial why those equations do not contain $E_{\perp}$ since the transverse component is not constrained from the Poisson equation. Note that the first term on the right hand side for ions is exactly the same as that for electrons Therefore, the contribution of ions to the charge density is 
non-negligible. On the other hand, the second term for ions is smaller by a factor of $\sqrt{m_{e} / m_{i}}$ than that for electrons. This is the essential point to make possible in-phase acoustic oscillations between electrons and ions. The space charge carried by ion acoustic oscillations can be canceled by electrons to maintain charge neutrality. In the case of a pure electron plasma examined by Ramani and Laval (1978), electrons have to keep charge neutrality by themselves. Therefore, the amplitude of the acoustic oscillation for an electron plasma has to be almost zero. The Poisson equation provides the relation between electric and magnetic fields as

$$
E_{k}=\frac{\epsilon \delta_{T}}{4} \frac{\sin \theta}{1+\left(k \lambda_{\mathrm{D}}\right)^{2}} \frac{v_{\text {th }, e}}{c} B_{z} .
$$

The term $\left(k \lambda_{\mathrm{D}}\right)^{2}$ in the denominator comes from the lefthand side of the Poisson equation and is always a very small number since the wavelength of the mode which we are interested in is much larger than the Debye length. We therefore neglect this term. This corresponds to the so-called plasma approximation (Chen, 1974; Tanaka and Nishikawa, 1996) and ensures that charge neutrality is kept to high accuracy even though the longitudinal oscillation exists. When the wave vector is parallel to the temperature gradient $(\theta=0)$, this equation implies $E_{k}=0$. The mode (1) becomes purely transverse. Compared with the case of pure electron oscillations as in Ramani and Laval (1978), the right-hand side of Eq. (28) is a factor of two smaller, which makes mode (1) more unstable as shown in below. No constraint comes from the Poisson equation for mode (2) since it is a purely transverse mode.

We are now in a position to derive the dispersion relation. Ampère's law is written as

$$
-i k B_{z}=\frac{4 \pi e}{c}\left(\left\langle v_{\perp} f_{\vec{k}, i}\right\rangle-\left\langle v_{\perp} f_{\vec{k}, e}\right\rangle\right)+\frac{\partial E_{\perp}}{c \partial t}
$$

for mode (1), and

$$
i k B_{\perp}=\frac{4 \pi e}{c}\left(\left\langle v_{z} f_{\vec{k}, i}\right\rangle-\left\langle v_{z} f_{\vec{k}, e}\right\rangle\right)+\frac{\partial E_{z}}{c \partial t}
$$

for mode (2). Under the low-frequency condition, the last terms on the right-hand side of Eqs. (29) and (30), which are the displacement current, are negligibly small. We thus neglect these terms. Further, the contribution of ions to the current density is the order of $\sqrt{m_{e} / m_{i}}$ smaller than that of electrons since the current density carried by each particle is proportional to the thermal velocity of each particle. Therefore, the current density carried by ions introduces only a negligible contribution to the dispersion relation. The dispersion relation for the real parts $\omega_{\mathrm{r}}$ and the imaginary parts $\omega_{\mathrm{i}}$ in leading order of $\epsilon$ is then obtained as follows:

$$
\begin{aligned}
\omega_{r}= & \frac{\epsilon \delta_{T}}{4} k v_{\mathrm{th}, e} \cos \theta \\
\omega_{i}= & \frac{\epsilon^{2} \delta_{T}^{2}}{4 \sqrt{\pi}} k v_{\mathrm{th}, e}\left(2 \cos ^{2} \theta-\sin ^{2} \theta\right) \\
& -\frac{1}{\sqrt{\pi}}\left(\frac{c}{\omega_{\mathrm{pe}}}\right)^{2} k^{3} v_{\mathrm{th}, e}
\end{aligned}
$$

for mode (1), and

$$
\omega_{r}=\frac{\epsilon \delta_{T}}{4} v_{\mathrm{th}, e} k \cos \theta
$$

$$
\omega_{i}=\frac{\epsilon^{2} \delta_{T}^{2}}{2 \sqrt{\pi}} v_{\mathrm{th}, e} k \cos ^{2} \theta-\frac{1}{\sqrt{\pi}}\left(\frac{c}{\omega_{\mathrm{pe}}}\right)^{2} v_{\mathrm{th}, e} k^{3},
$$

for mode (2).

Since the imaginary part $\omega_{\mathrm{i}}$ is of order $\epsilon^{2}$, we have checked whether the leading order of the dispersion relation is changed when the second-order distribution functions $f_{e}^{(2)}$ and $f_{i}^{(2)}$ are taken into consideration. We have confirmed that these second-order distribution functions only introduce one order higher terms both in real and imaginary parts, and thus the main results shown above are not changed.

\section{Instability and Mode Characteristics}

The dispersion relations (31), (32), (33), and (34) show that the low-frequency mode, for which the phase velocity of the wave is slower than the ion thermal velocity, can exist as long as $\epsilon<\sqrt{m_{e} / m_{i}} \sim 0.025$ is satisfied. This is the first confirmation that the Ramani and Laval (1978) type instability can exist beyond the wall of square root of the mass ratio. The dispersion relations are almost the same as those obtained for the electron plasma by Ramani and Laval (1978). However, there is a slight difference in the imaginary part of mode (1). The difference comes from the difference in the charge neutrality condition (28). As explained in Section 3, in our case, an in-phase acoustic oscillations between electrons and ions is possible. Hence, the space charge carried by ion acoustic oscillations can be canceled by electrons to maintain charge neutrality. However, in the case of the pure electron plasma examined by Ramani and Laval (1978), electrons have to keep charge neutrality by themselves. Therefore, the amplitude of acoustic oscillations for the electron plasma has to be almost zero.

The characteristics of the instability are summarized as follows. For mode (1), the imaginary part of the wave frequency is positive, and the instability sets in when the direction of the wave vector is within the double cone spanned by $\theta \in\left[-\theta_{\mathrm{cr}}, \theta_{\mathrm{cr}}\right]$ and $\theta \in\left[\pi-\theta_{\mathrm{cr}}, \pi+\theta_{\mathrm{cr}}\right]$, where $\theta_{\mathrm{cr}} \equiv$ $\arccos (1 / \sqrt{3})\left(0 \leq \theta_{\text {cr }} \leq \pi / 2\right)$. For comparison, we recalculated the dispersion relations for pure electron plasma. In the case of a pure electron plasma, a factor of 2 appears in front of the $\sin ^{2} \theta$ term in the imaginary part (this result is slightly different from the Ramani and Laval (1978) result). Therefore, the unstable region in $\vec{k}$-space in the case of a pure electron plasma is somewhat narrower than in our case. It shows that acoustic oscillations of ions assist the instability. For mode (2), the unstable mode exists for all direction of the wave vector, although the growth rate decreases as $\theta$ increase from 0 to $\pi / 2$ for fixed $k$. Two modes get identical when $\theta=0$. For both modes, the growth rate is maximum when $\theta=0$. Since the imaginary part of the wave frequency is a third order polynomial in $k$ with negative coefficient for $k^{3}$, there exists a maximum growth rate $\omega_{i, \max }$. For mode (1), $\omega_{i, \max }$ is given as

$$
\omega_{\mathrm{i}, \max } \sim \frac{\epsilon^{3} \delta_{T}^{3}}{12 \sqrt{3 \pi}} \frac{v_{\mathrm{th}, e}}{c} \omega_{\mathrm{pe}}\left|3 \cos ^{2} \theta-1\right|^{3 / 2},
$$

when

$$
k=k_{\max }=\frac{\epsilon \delta_{T}}{2 \sqrt{3}} \frac{\omega_{\mathrm{pe}}}{c}\left|3 \cos ^{2} \theta-1\right|^{1 / 2},
$$


for $\theta \in\left[-\theta_{\mathrm{cr}}, \theta_{\mathrm{cr}}\right]$ or $\theta \in\left[\pi-\theta_{\mathrm{cr}}, \pi+\theta_{\mathrm{cr}}\right]$. For mode (2),

$$
\omega_{\mathrm{i}, \max } \sim \frac{\epsilon^{3} \delta_{T}^{3}}{3 \sqrt{6 \pi}} \frac{v_{\mathrm{th}, e}}{c} \omega_{\mathrm{pe}}\left|\cos ^{3} \theta\right|
$$

when

$$
k=k_{\max }=\frac{\epsilon \delta_{T}}{\sqrt{6}} \frac{\omega_{\mathrm{pe}}}{c}|\cos \theta|,
$$

for arbitrary $\theta$. For the wave vector which satisfies the above conditions, the real part $\omega_{\mathrm{r}}$ of the wave frequency is obtained as

$$
\omega_{\mathrm{r}} \sim \frac{\epsilon^{2} \delta_{T}^{2}}{8 \sqrt{3}} \frac{v_{\mathrm{th}, e}}{c} \omega_{\mathrm{pe}}\left|3 \cos ^{2} \theta-1\right|^{1 / 2} \cos \theta
$$

for mode (1), and

$$
\omega_{\mathrm{r}} \sim \frac{1}{4 \sqrt{6}} \epsilon^{2} \delta_{T}^{2} \frac{v_{\mathrm{th}, e}}{c} \omega_{\mathrm{pe}} \cos ^{2} \theta
$$

for mode (2).

The electric field strength can be related to the magnetic field strength. For mode (1),

$$
\left|E_{k}\right| \sim \frac{\epsilon \delta_{T}}{4} \frac{v_{\text {th }, e}}{c}\left|\vec{B}_{\vec{k}}\right||\sin \theta| .
$$

When $k=k_{\max }$, the electric field perpendicular to the $\vec{k}$ direction is related to the magnetic field as

$$
\left|E_{\perp}\right| \sim \frac{\epsilon \delta_{T}}{4} \frac{v_{\text {th }, e}}{c}\left|\vec{B}_{\vec{k}}\right||\cos \theta| .
$$

For mode (2),

$$
\left|E_{z}\right| \sim \frac{\epsilon \delta_{T}}{4} \frac{v_{\mathrm{th}, e}}{c}\left|\vec{B}_{\vec{k}}\right||\cos \theta|,
$$

when $k=k_{\max }$.
The electric field strength is then the order of $\epsilon$ smaller than the magnetic field strength. This nature combined with the low-frequency nature of the mode shows that the mode has similarity with a magneto-hydrodynamic mode. However, in the magneto-hydrodynamic treatment the mode and the instability cannot be identified. The instability is microscopic nature, in which case the resonance of particles with waves is essential. Therefore, the instability creates and amplifies the magnetic field without any seed field. It provides a new possibility for the origin of the cosmic magnetic field.

Clarifying the non-linear saturation level of the waves is one of the important next steps. A magnetic reconnection may play some role on determining the saturation level.

\section{References}

Chapman, S. and T. G. Cowling, The Mathematical theory of non-uniform gases, Cambridge University Press, Cambridge, 1960.

Chen, F. F., Introduction to Plasma Physics, 1974.

Fried, B. D., Mechanism for instability of transverse plasma waves, Phys Fuild., 2, 337, 1959

Hattori, M. and K. Umetsu, A possible route to spontaneous reduction of the heat conductivity by a temperature gradient-driven instability in electronion plasmas, ApJ, 533, 84, 2000.

Ikebe, Y., K. Makishima, Y. Fukazawa, T. Tamura, H. Xu, T. Ohashi, and K. Matsushita, Two-phase intracluster medium in the Centaurus cluster of galaxies, ApJ, 525, 581, 1999.

Melrose, D. B., Instabilities in space and laboratory plasmas, Cambridge Univ. Press, Cambridge, 1986.

Ramani, A. and G. Laval, Heat flux reduction by electromagnetic instabilities, Phys. Fluid., 21(6), 980, 1978.

Tanaka, M. and K. Nishikawa, Physics of high temperature plasmas, Maruzen, Tokyo, 1996.

Weibel, E. S., Spontaneously growing transverse wave in a plasma due to an anisotropic velocity distribution, Phys. Rev. Lett., 2, 83, 1959.

M. Hattori (e-mail: hattori@ astr.tohoku.ac.jp) and K. Umetsu 